\title{
Hiperparatiroidismo Primario: Reporte de caso clínico
}

\author{
Primary Hyperparathyroidism: Case report
}

\author{
Onix Arita Melzer, ${ }^{1}$ Manuel Maldonado, ${ }^{2}$ Reinaldo Castro, ${ }^{3}$ \\ César Alas-Pineda, ${ }^{4}$ Fanny Ponce-Barahona, ${ }^{5}$ Irina Álvarez-Arita. ${ }^{6}$
}

\author{
${ }^{1}$ Médico Especialista en Medicina Interna y Sub-especialista en Endocrinología, Hospital Bendaña; \\ ${ }^{2}$ Médico Especialista en Cirugía Oncológica, Liga Contra el Cáncer de Honduras; \\ ${ }^{3}$ Médico Especialista en Patología, Hospital del Valle; \\ ${ }^{4}$ Estudiante de Sexto Año de la Carrera de Medicina, Facultad de Medicina y Cirugía, Universidad Católica de Honduras; \\ ${ }^{5}$ Estudiante de Quinto Año de la Carrera de Medicina, Facultad de Medicina y Cirugía, Universidad Católica de Honduras; \\ ${ }^{6}$ Médico General, Hospital Bendaña; San Pedro Sula, Honduras.
}

\begin{abstract}
RESUMEN. Antecedentes: El hiperparatiroidismo primario, tercer trastorno metabólico más común a nivel mundial, provoca un aumento del calcio sérico y de la hormona paratiroidea, causado por una hiperactividad de las glándulas paratiroideas. En el $85 \%$ de los casos, se debe a un adenoma paratiroideo. Es predominantemente asintomático (>80\%), pero sus manifestaciones pueden ser musculo-esqueléticas, neurológicas, psiquiátricas, renales, cardiovasculares, y gastrointestinales. Descripción del Caso Clínico: Paciente femenina de 53 años de edad, con antecedente de hipercolesterolemia familiar heterocigoto e hipertensión arterial; presenta insomnio, náuseas, pérdida de peso, mialgias, astenia, reflujo gastroesofágico e irritabilidad. Ante indicación de exámenes de laboratorio muestra hipercalcemia leve. Se comprueba un adenoma paratiroideo derecho y nódulos tiroideos coloides benignos en lóbulo izquierdo mediante gammagrafía Tc99m MIBI, siendo sometida a su resección con posterior evolución satisfactoria. Conclusiones: Considerando la predilección asintomática del hiperparatiroidismo primario y su preferencia de presentación como adenoma, resulta indispensable su sospecha ante toda hipercalcemia. Requiere entonces una concientización amplia no solo en el ámbito médico sino también en la comunidad, ampliando esfuerzos entre el clínico, cirujano, y patólogo. Se recomienda medir el calcio y fósforo sérico de forma rutinaria en la evaluación clínica cotidiana de cada paciente para su detección.
\end{abstract}

Palabras clave: Cintigrafía, Glándulas paratiroides, Hipercalcemia, Hiperparatiroidismo primario, Neoplasias de las paratiroides.

\section{INTRODUCCIÓN}

El hiperparatiroidismo primario (HPTP) es el tercer trastorno metabólico más común, provocado por un aumento de la secreción de hormona paratiroidea (PTH) por una o más glándulas paratiroides, resultando en hipercalcemia, y con menor frecuencia hipofosfatemia. ${ }^{1,2}$ Estados Unidos presenta una prevalencia de 1 a 2 casos cada 1,000 personas $(0.1 \%)$ y una incidencia de $70,000-90,000$ anualmente. $^{3}$ Se presenta con más frecuencia entre la cuarta y sexta década de la vida, predominando en mujeres con una relación 3:1. ${ }^{1}$ En ancianos, tiene una prevalencia de 1 en cada 100 casos, y en niños y jóvenes se presenta como componente de Neoplasia Endocrina Múltiple (NEM) tipo 1 y 2. A pesar de que su etiología permanece desconocida, se considera la exposición a la radiación en el cuello, especialmente durante la niñez como el principal factor de riesgo. Se ha evidenciado también la presencia de un componente genético en casos de adenoma e hiperplasia multiglandular. ${ }^{4}$ El $80 \%$ de los casos de HPTP son causados por un adenoma paratiroideo úni-

Recibido: 25-1-2019; Aceptado para publicación 30-6-2019

Dirección para correspondencia: Dr. Onix Arita Melzer,

Correo electrónico: onixarita@yahoo.com

Conflictos de interés. Los autores declaran no tener conflictos de interés en relación a este artículo.

DOI: https://doi.org/10.5377/rmh.v87i2.11905 co, el 15 a $20 \%$ por hiperplasia glandular, $4 \%$ por un adenoma múltiple y $0.5-1 \%$ por carcinoma de paratiroide. ${ }^{5}$

El diagnóstico de HPTP en pacientes asintomáticos ha aumentado considerablemente desde el año 1960, debido a una mayor accesibilidad a exámenes de laboratorio de rutina. ${ }^{3,6,7} \mathrm{En}$ el adenoma paratiroideo, la hipercalcemia suele ser discreta (1 $\mathrm{mg} / \mathrm{dl}$ sobre el valor normal), presentándose entre 10.0 y 11.0 $\mathrm{mg} / \mathrm{dl}{ }^{8}$ También puede presentarse una elevación de la hormona paratiroidea, fosfatasa alcalina y de la excreción urinaria de calcio (aumentada en un 25 a $35 \%$ ). ${ }^{6}$ Las manifestaciones clínicas aparecen de forma tardía en la evolución de HPTP, incluyendo manifestaciones esqueléticas (dolor óseo, fracturas, crecimiento anormal), renales (nefrolitiasis y lesión renal), neuromusculares, cardiovasculares y digestivas. Las fracturas más comunes son de huesos largos, clavículas, costillas y pelvis. Se ha reportado la aparición de tumor pardo en clavículas, costillas, pelvis, fémur y huesos faciales como una lesión lítica detectable en radiografía y gammagrafía en un 3\% de los casos. Las manifestaciones gastrointestinales incluyen estreñimiento (33\%), pirosis $(24 \%)$, náuseas $(24 \%)$, pérdida del apetito $(15 \%)$ y dolor abdominal inespecífico $(29 \%){ }^{9}$

Los estudios de imagen proveen un diagnóstico definitivo ante datos laboratoriales alterados. Dado al pequeño tamaño de las glándulas paratiroides y su estructura parecida al de la 
glándula tiroides, la ecosonografía es de escaso a nulo uso. ${ }^{4}$ El estudio de medicina nuclear, cintigrafía con tecnecio $99 \mathrm{~m}$ MIBI (methoxyisobutylisonitril) es el procedimiento de elección para localizar glándulas paratiroides hiperactivas. Junto a una ecografía de partes blandas de cuello, su sensibilidad aumenta hasta un $96 \%$ para la detección de adenomas solitarios. ${ }^{6,10,11}$ El estudio MIBI-SPECT (tomografía computarizada de emisión monofotónica) se indica solamente en casos de enfermedad recurrente, con una sensibilidad de $46-86 \%$ para adenomas de difícil localización (usualmente mediastinales), enfermedad multiglandular y múltiples adenomas. ${ }^{6}$ Como última opción, se indica la resonancia magnética. ${ }^{8}$

El tratamiento de consenso es la paratiroidectomía con hemitiroidectomía ipsilateral, alcanzando una tasa de curación de 95-98\% ${ }^{4,9}$ Se indica en pacientes con hiperparatiroidismo sintomático, o en asintomáticos menores de 50 años con un aumento del calcio sérico superior a $1 \mathrm{mg} / \mathrm{dl}$ sobre su valor normal, o con densidad mineral ósea igual o menor a menos (-) 2.5 de T score en cualquier parte. ${ }^{8}$ Se realiza un monitoreo transoperatorio de la HPT. Su disminución en un 50\%, así como la hipocalcemia transitoria se consideran indicadores de curación. ${ }^{1}$ La paratiroidectomía posee una morbilidad de $1-2 \%$, siendo sus principales complicaciones la lesión del nervio laríngeo recurrente, formación de hematomas, hipocalcemia permanente e hipoparatiroidismo. Se recomienda sustituir una exploración cervical bilateral por una cirugía de mínima invasión debido a un menor riesgo de lesión al nervio laríngeo recurrente y de hipoparatiroidismo. ${ }^{4}$ Dentro de los beneficios de una cirugía de mínima invasión están corta estancia hospitalaria y menos días de recuperación. ${ }^{6}$

\section{CASO CLÍNICO}

Se reporta caso de paciente femenina de 53 años de edad, hondureña, quien acude a consulta externa de endocrinología presentando insomnio, náuseas, pérdida de peso, mialgias, astenia, reflujo gastroesofágico, e irritabilidad de 6 meses de evolución. Refiere antecedentes heredofamiliares de hipertensión arterial en madre y dislipidemia mixta e hígado graso en padre.

Refiere antecedentes personales patológicos de hipercolesterolemia familiar heterocigota tratada con $40 \mathrm{mg}$ de atorvastatina diarios e hipertensión arterial tratada con $16 \mathrm{mg}$ de candersartán y $12.5 \mathrm{mg}$ de hidroclorotiazida diarios. A la exploración física se encontró un peso de 72.7 kilogramos, talla 1.60 metros, índice de masa corporal $28.4 \mathrm{~kg} / \mathrm{m}^{2}$, frecuencia cardiaca 80 latidos por minuto, presión arterial 160/90 mmHg. La biometría hemática y química sanguínea reportó los siguientes valores: Glóbulos blancos 8650/uL; Hemoglobina 14.70 g/dl; Plaquetas 238000/uL; Glucosa en ayunas $94 \mathrm{mg} / \mathrm{dl}$; Ácido úrico 5.6 $\mathrm{mg} / \mathrm{dl}$; Creatinina $0.9 \mathrm{mg} / \mathrm{dl} ;$ BUN $10 \mathrm{mg} / \mathrm{dl}$; TGO 31 u/L; TGP 49 u/L; Triglicéridos $139 \mathrm{mg} / \mathrm{dl}$; Colesterol total $291 \mathrm{mg} / \mathrm{dl}$; HDL 52 $\mathrm{mg} / \mathrm{dl}$; LDL $211 \mathrm{mg} / \mathrm{dl}$; Calcio $10.67 \mathrm{mg} / \mathrm{dl}$; Calcio iónico $5.9 \mathrm{mg} /$ dl; Magnesio $2.1 \mathrm{mg} / \mathrm{dl}$; Fósforo: $3.6 \mathrm{mg} / \mathrm{dl}$; T3 $0.78 \mathrm{ng} / \mathrm{mL}$; T4 $6.51 \mathrm{mcg} / \mathrm{dL} ; \mathrm{TSH} 0.93 \mathrm{mUl} / \mathrm{mL}$; Calcio en orina de $24 \mathrm{~h} 409.3$ mg; PTH 102.8 pg/ml. Se hace el diagnóstico clínico de hiperparatiroidismo primario basado en los niveles aumentados de calcio total, calcio iónico, calcio urinario en 24 horas, y hormona paratiroidea, sospechándose como primera posibilidad etiológica un adenoma paratiroideo, solicitándose subsecuentemente una ultrasonografía de cuello y un cintigrama de paratiroides con Tc99m MIBI.

El ultrasonido de cuello reveló una masa compatible con adenoma paratiroideo derecho y nódulos tiroideos de aspecto benigno, indicándose biopsia por aspiración con aguja fina (BAAF) para el de mayor tamaño en lóbulo tiroideo izquierdo, el cual confirmó un nódulo coloide benigno negativo para malignidad. El estudio de medicina nuclear gammagráfico de paratiroides con Tc99m MIBI (Figura 1) reveló captación en lóbulo tiroideo derecho compatible con adenoma paratiroideo y captación irregular en lóbulo tiroideo izquierdo sugiriendo nódulos tiroideos.

La paciente fue intervenida quirúrgicamente para resección de glándula paratiroides derecha con estudio transoperatorio por congelación y hemitiroidectomía izquierda. A la intervención, la glándula paratiroidea derecha presenta consistencia dura en comparación al tejido circunvecino y al resto de glándulas de la paciente, con un tamaño de $2 \mathrm{~cm}$ de diámetro (Figura 2). El estudio anatomopatológico confirma adenoma de tipo folicular de glándula paratiroides derecha (Figura 3), con cortes histo-
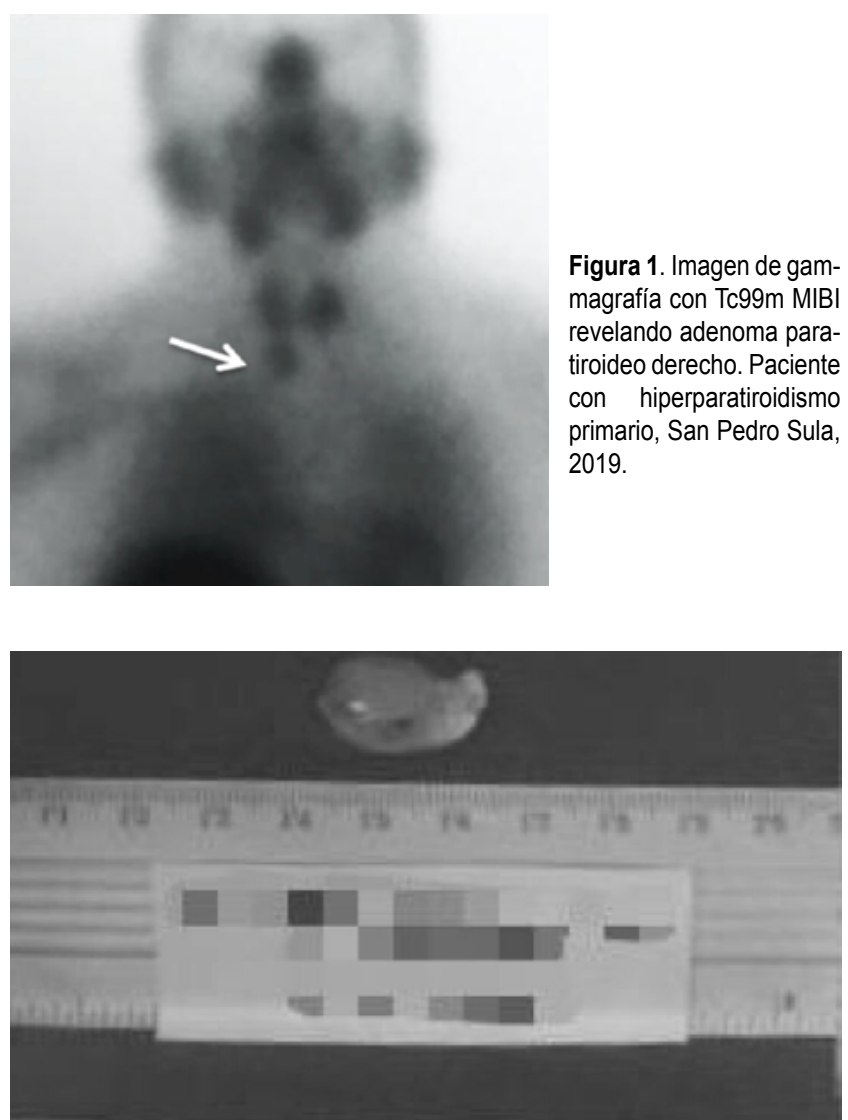

Figura 2. Pieza quirúrgica demostrando adenoma paratiroideo de glándula inferior derecha. Paciente con hiperparatiroidismo primario, San Pedro Sula, 2019. 


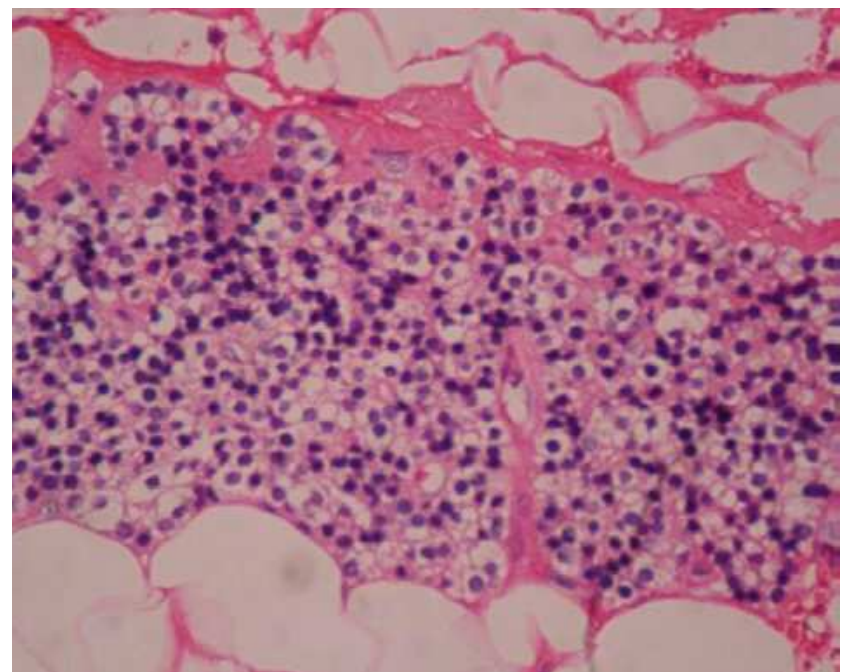

Figura 3. Imagen histopatológica demostrando bandas de tejido paratiroideo con células poligonales uniformes, núcleos centrales pequeños, sin atipias, con tendencia a la formación de folículos. Hematoxilina - Eosina, 40x. Paciente con hiperparatiroidismo primario, San Pedro Sula, 2019.

lógicos revelando células principales poligonales uniformes con núcleos centrales pequeños sin atipias, citoplasma amplio claro y bordes bien definidos en el $90 \%$ de la glándula. Algunas células muestran tendencia a la formación de folículos. De manera periférica se identifican bandas de tejido paratiroideo dentro de los límites normales, la lesión se encuentra delimitada por una delgada capa de tejido fibroconectivo, sin evidencia de malignidad. La resección de la porción afectada del lóbulo tiroideo izquierdo revela cambios bociogénicos negativos por malignidad.

La paciente fue egresada con evolución satisfactoria, con calcio sérico a las $24 \mathrm{hrs}, 48 \mathrm{hrs}$ y $72 \mathrm{hrs}$ de $9.5 \mathrm{mg} / \mathrm{dl}, 10.2 \mathrm{mg} /$ dl y $9 \mathrm{mg} / \mathrm{dl}$ respectivamente, manteniendo calcemias normales en el postoperatorio. Actualmente, conserva terapia sustitutiva del hipotiroidismo preexistente de $88 \mathrm{mcg}$ diarios de levotiroxina en ayunas, $40 \mathrm{mg}$ de atorvastatina y $16 / 12.5 \mathrm{mg}$ candesartán/ hidroclorotiazida diarios.

\section{DISCUSIÓN}

Resulta contradictorio como el HPTP es uno de los trastornos endocrinológicos más comunes y a la vez es un desafío diagnosticar clínicamente. De naturaleza asintomática (>80\%), su diagnóstico se basa en una alteración de laboratorio reflejada como un aumento leve del calcio sérico $(1 \mathrm{mg} / \mathrm{dl}$ sobre el valor normal. ${ }^{1,9,11}$ La paciente del caso presentó manifestaciones musculoesqueléticas, neurológicas, gastrointestinales y cardíacas, en consonancia a las mencionadas en la literatura. ${ }^{12}$ Las manifestaciones gastrointestinales suelen ser pasadas por alto al presentarse de forma aislada, por lo que se incita a una medición de calcio sérico ante todo dolor abdominal inespecífico. ${ }^{9}$ Sin embargo, el HPTP también puede ser una urgencia médica al presentarse como una crisis paratiroidea, con hipercalcemia severa (>15 mg/dl) y síntomas graves, principalmente neurológicos, usualmente causados por neoplasias y carcinomas., ${ }^{1,13}$ La hipercalcemia debida a adenoma paratiroideo suele ser leve y de evolución crónica. ${ }^{4}$ Se resalta la exposición a radiación de cuello especialmente durante la infancia, como principal factor de riesgo para el desarrollo de HPTP. ${ }^{14}$

Siendo el adenoma paratiroideo la principal causa de HPTP, se indica la realización de ultrasonido en conjunto con estudio de medicina nuclear (Tc99m MIBI), para la localización más sensible de un adenoma. ${ }^{15}$ El diagnóstico diferencial entre el adenoma y carcinoma paratiroideo ( $1 \%$ de casos de HPTP) puede incluir la presencia de ciertas proteínas, pero el diagnóstico definitivo lo determina los estudios de imagen. La Ki-27 se reporta con mayor frecuencia en carcinoma (27\%) en comparación a adenoma (2\%), y la proteína de retinoblastoma se presenta con mayor frecuencia en adenoma. ${ }^{16,17}$

Los adenomas paratiroideos suelen ser menores de $2 \mathrm{~cm}$ y no palpables. Mayor a este diámetro se suele tratar de carcinomas o adenomas en glándulas ectópicas $(9 \%)$, usualmente en el mediastino superior. Sin embargo, se han reportado casos de adenomas gigantes de paratiroides (10\%), con mayor mortalidad. A mayor peso del adenoma, aumenta la severidad de síntomas, crisis paratiroideas y mortalidad. A su vez, la relación entre el nivel de calcio sérico y el peso del adenoma es directamente proporcional. En pacientes con hipercalcemia $>15 \mathrm{mg} /$ $\mathrm{dl}$ el peso medio del adenoma es de $4.9 \mathrm{~g}$, y en pacientes con calcio sérico $<15 \mathrm{mg} / \mathrm{dl}$, la media es de $2.1 \mathrm{~g}$. ${ }^{1}$

La paratiroidectomía es el tratamiento de elección para adenoma de paratiroides, obteniendo una cifra de curación superior al 95\%., ${ }^{2,7}$ En un estudio retrospectivo de 1402 pacientes, se reportó una tasa de curación del $98.4 \%$, con recurrencias siendo más frecuente en casos de doble adenoma (9\%). ${ }^{2}$ La complejidad de la cirugía dependerá del número de glándulas afectadas y la localización del adenoma. ${ }^{1}$ Las técnicas de la cirugía han evolucionado mucho en los últimos años, implementándose técnicas de mínima invasión, medición de PTH transoperatoria, y biopsia por congelación para disminuir la morbimortalidad, estancia intrahospitalaria, y tiempo operatorio., 48,19 Sus complicaciones más frecuentes incluyen la hipocalcemia transitoria y el hipoparatiroidismo permanente. ${ }^{7}$ El hipoparatiroidismo se presenta por la alteración de la vascularización de las glándulas paratiroides y por la biopsia de glándulas paratiroides normales. ${ }^{4}$ La hipocalcemia transitoria (días a semanas) se debe a una hipotrofia de glándulas sanas y es considerado como un indicador de curación. ${ }^{1}$ En caso de la persistencia de calcemias postoperatorias normales, se debe sospechar de enfermedad subyacente no diagnosticada o de persistencia in situ de fragmentos de paratiroides resecadas. ${ }^{10}$ En pacientes asintomáticos o no candidatos para la cirugía, se puede utilizar la terapia farmacológica con cinacalcet, manteniendo una vigilancia cercana de la evolución de los niveles de calcio sérico y PTH en el paciente. ${ }^{2,5}$ 
Considerando la predilección asintomática del HPTP y su preferencia de presentación como adenoma, resulta indispensable sospechar de él ante toda hipercalcemia y crear concientización. Se recomienda realizar mediciones de rutina de calcio y fósforo sérico como parte de la evaluación clínica cotidiana de cada paciente, habilitando así su detección. Simultáneamente, dado a lo poco que se conoce sobre la etiología del adenoma paratiroideo, se debe plantear la posibilidad de que éste forme parte de un síndrome. ${ }^{7}$

\section{CONTRIBUCIONES}

OAM realizó el diagnóstico del caso, dirigió tratamiento médico y refirió a cirujano oncólogo para tratamiento quirúrgico. MM realizó la resección quirúrgica del adenoma paratiroideo. $\mathrm{RC}$ realizó el diagnostico histopatológico del adenoma paratiroideo resecado y fue negativo por malignidad. CAP y FPB contribuyeron con la revisión bibliográfica y redacción del marco teórico del tema en revisión. IAA acompañó el manejo clínico y postoperatorio del caso. IAA y FPB lideraron la redacción del artículo con la contribución de todos los autores. Todos los autores atendieron las recomendaciones editoriales y aprobaron la versión final del artículo.

\section{REFERENCIAS}

1. Cuenca D, Peña JF, Mercado M. Adenoma gigante de paratiroides: presentación de un caso. Rev Endocrinol Nutr. 2012; 20(2): 88-91.

2. Restrepo Valencia CA, Cáceres Escobar D. Doble adenoma de paratiroides como presentación de hiperparatiroidismo primario y detalles de su tratamiento. Act Med Col. 2016; 41(3): 198-201.

3. Mazeh H, Kouniavsky G, Schneider DF, Makris KI, Sippel RS, Dackiw AP et al. Intrathyroidal parathyroid glands: small, but mighty (a Napoleon phenomenon). Surg. 2012; 152(6):1193-200.

4. Olivera Fajardo D, Puerto Lorenzo JA, Torres Ajá L. Adenoma paratiroideo. Presentación de un caso. 2016; 14(5):567-572.

5. Mackenzie Feder J, Sirrs S, Anderson D, Sharif J, Khan A. Primary hyperparathyroidism: an overview. Int J Endocrinol. 2011;2011: 251410.

6. Solís Torres J, Mora-Segura E. Hiperparatiroidismo primario por adenoma paratiroideo mediastinal. Act Med Cost. 2015; 57(2): 86-87.

7. Khan A, Grey A, Shoback D. Medical management of asymptomatic primary hyperparathyroidism: proceedings of the third international workshop. J Clin Endocrinol Metab. 2009; 94(2):373-381.

8. Granadas Canseco E, Servín Torres E, Piscil Salazar MA, Velázquez García JA, Serrano Galeana I, Granados Canseco R et al. Experiencia quirúrgica en el hiperparatiroidismo primario en el Hospital de Especialidades del Centro Médico Nacional "La Raza". Cir Gen. 2012; 34(4): 249-253.

9. Stoopen-Margain E, Valanci-Aroesty S, Castaneda-Martínez L, BaqueraHeredia J, Sainz-Hernández JC. Enfermedad paratiroidea: el espectro completo, de adenoma a carcinoma. Reporte de 3 casos. Cir Cir. 2017; 85(6):549-551.

10. Rocha L, Suazo C, González M, Lee K, Rossel G. Hiperparatiroidismo primario y cáncer de paratiroides: Caso clínico. Rev Chil Cir. 2010; 62(5):512515.

11. Goñi El, Campusano MC, Gejman ER, Pilar OB. Hiperparatiroidismo primario de origen multiglandular con glándula paratiroides intratiroídea hi- perfuncionante. Rev Chil Cir. 2011; 63(4): 418-421.

12. Khan A, Grey A, Schoback D. Medical management of asymptomatic primary hyperparathyrodism: proceedings of the Third International Workshop. J Clin Endocrinol Metab. 2009; 94(2): 373-381.

13. Irún AP, Santamaría AG, Garmilla NF, Barquín SA. El adenoma paratiroideo también puede ser una urgencia. SEMERGEN-Medicina de Familia [Internet]. 2011 [consultado 10 de diciembre 2018];37(2): 99-101. Disponible en: http://linkinghub.elsevier.com/retrieve/pii/S1138359310004107?via=sd.

14. Davoli F, Rena O, Pirondini E, Casadio C, Monza I. Quiste paratiroideo funcionante gigante de localización mediastínica: una causa insólita de disnea de esfuerzo y disfagia de carácter leve. Arch de Bronconeumol [Internet]. 2013 [consultado 11 de diciembre 2018];49(9):408-409. Disponible en: http://www.archbronconeumol.org/es/giant-functioning-mediastinalparathyroid-yst/articulo/90224089/

15. Taieb D, Hindie E, Grassetto G, Colletti P, Rubello D. Parathyroid Scintigraphy When, How, and Why? A Concise Systematic Review. Clint Nucl Med. 2012;37(6):568-574.

16. Stojadinovic A, Hoos A, Nissan A, Dudas M, Cordon-Cardo C, Shaha A, et al. Parathyroid neoplasms: clinical, histopathological, and tissue microarray-based molecular analysis. Hum Pathol 2003; 34(1): 54-64.

17. Cetani F, Pardi E, Viacava P, Pollina GD, Fanelli G, Picone A et al. A reappraisal of the $\mathrm{Rb} 1$ gene abnormalities in the diagnosis of parathyroid cancer. Clin Endocrinol (Oxf). $2004 ; 60$ (1):99-106.

18. Kunstman JW, Udelsman R. Superiority of minimally invasive parathyroidectomy. Adv Surg. 2012;46:171-489.

19. Barczynski M, Konturek A, Hubalewska-Dydejczyk A, Cichon S, Nowak W. Evaluation of Halle, Miami, Rome, and Vienna intraoperative iPTH assay criteria in guiding minimally invasive parathyroidectomy. Langenbecks Arch Surg. 2009;394(5):843-849.

ABSTRACT. Background: Primary hyperparathyroidism, third most common metabolic disorder worldwide, provokes an increase of serum calcium and parathyroid hormone caused by a hyperactivity of parathyroid glands. In $85 \%$ of cases, it is due to a parathyroid adenoma. Predominantly asymptomatic $(>80 \%)$, its manifestations can be musculoskeletal, neurologic, psychiatric, renal, cardiovascular and gastrointestinal. Clinical case description: 53-year-old female with history of heterozygous familial hypercholesterolemia and arterial hypertension, presents insomnia, nausea, weight loss, myalgia, asthenia, gastroesophageal reflux, and irritability. Further exams show hypercalcemia. A right parathyroid gland adenoma is presented through Tc99-sestamibi scintigraphy, along with left benign thyroid nodules. Patient undergoes parathyroidectomy, with posterior satisfactory evolution. Conclusions: Considering primary hyperparathyroidism's asymptomatic nature predilection and its preference for adenoma presentation, it is necessary to suspect it when hypercalcemia is present. Awareness is necessary not only in the medical field, but also in the general community through conjoint efforts between the clinical, surgeon, and pathologists. Routine calcium and phosphorus measurements as part of every patient's scheduled clinical evaluation are strongly suggested in order to enable its detection.

Keywords: Hypercalcemia; Hyperparathyroidism, primary; Parathyroid glands; Parathyroid neoplasms; Radionuclide imaging. 